\title{
Dynamics of the Galactic Bulge
}

\author{
TIM DE ZEEUW
}

Sterrewacht Leiden

\begin{abstract}
Recent work on the intrinsic shape, the internal kinematics of stars and gas, and the dynamics of the Galactic Bulge is discussed. Starcounts, measurements of the integrated light and the kinematics of the atomic and molecular gas all provide strong evidence that the Bulge is triaxial, and is rotating fairly rapidly. To date, there is little evidence for triaxiality in the stellar kinematics: the available stellar velocities are consistent with Kent's (1992) oblate model. This unsatisfactory situation is expected to improve rapidly.
\end{abstract}

\section{Introduction}

The Galactic Bulge is one of the major components of the Galaxy. A careful study of its morphology, kinematic and dynamical properties is required to answer questions such as: Can we think of the Bulge as a small elliptical galaxy? Was it formed before or after the disk? What is its relation to the metal-poor stellar halo, and to the dark halo? An extensive discussion of these and related issues was given by Freeman (1987), Gilmore, King \& van der Kruit (1990), and Spergel (1992). Here we concentrate on two specific areas of research on the Galactic Bulge, namely, i) what is its intrinsic shape, and ii) what is its internal velocity structure? We first consider measurements of the integrated light and starcounts, as well as observations of the gaseous and the stellar kinematics, then discuss the dynamics of the Bulge, and finally compare the results briefly with what we know about the dynamics of bulges of other disk galaxies. Detailed discussions of the properties of other bulges, and of the relation between bulges and elliptical galaxies, are given by Kormendy and Franx, respectively, elsewhere in this volume.

\section{Morphology}

\subsection{INTEGRATED LIGHT}

The Bulge is heavily obscured at optical wavelengths, and is best studied in the infrared. Early measurements showed that the integrated surface brightness distribution between 2 and 2.4 micron is flattened, and has a central cusp (Becklin \& Neugebauer 1968; Matsumoto et al. 1982). A variety of functions have been proposed as fits to the observed surface brightness profile, including power laws, exponentials and even de Vaucouleurs' profiles. Sellwood \& Sanders (1988) reviewed much of the early work, and showed that the volume emissivity profile between 0.5 and $500 \mathrm{pc}$ from the Galactic Center is a power-law with slope -1.8, while at larger radii the profile steepens considerably, and the slope approaches -3.7 . Kent (1992) analyzed the Spacelab 2.4 micron data (Kent, Dame \& Fazio 1991) and showed that foreground contamination by the disk amounts to $40 \%$ of the observed light. The corrected profile (Fig. 1) is similar to the one proposed by Sellwood \& Sanders, has an inner slope of -1.85 , and a minor axis scale-length of $\sim 400$ pc. There is no component with a scale-length of $2.7 \mathrm{kpc}$, as is sometimes claimed. 


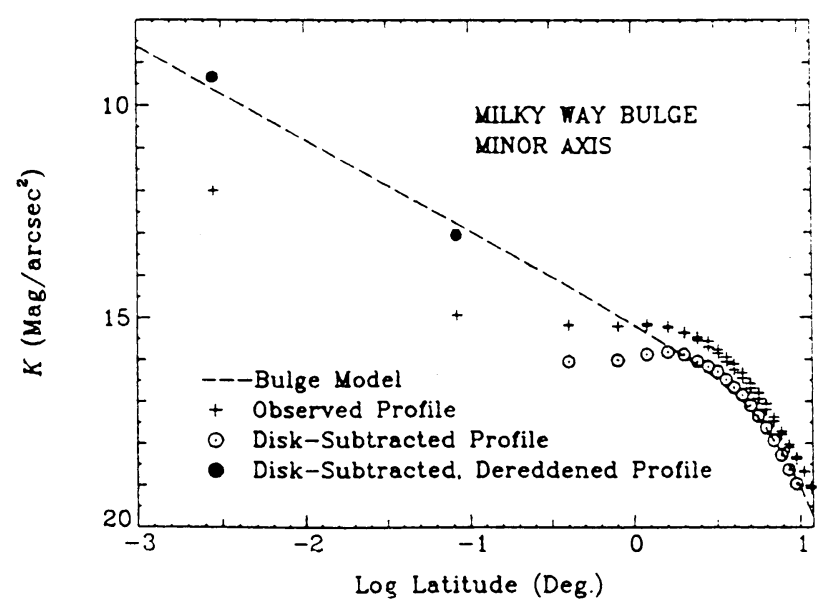

Fig. 1. Minor axis surface brightness profile of the Bulge in the near-infrared, as given by Kent (1992).

Most previous studies have assumed that the Bulge is oblate, with its short axis perpendicular to the Galactic plane. This means we observe the Bulge edge-on, and the surface brightness distribution can be deprojected uniquely to give the three-dimensional luminosity distribution (Rybicki 1987). Kent (1992) finds that the Bulge has an axis ratio of 0.61 , and is slightly box- or peanut-shaped. Inside one kpc the Bulge may be more flattened.

Blitz \& Spergel (1991b) reanalyzed the balloon measurements of Matsumoto et al. (1982), and found that the Bulge is in fact brighter at positive galactic longitude $\ell$ than at negative longitude. They argued that this is not due to extinction variations, but is caused by the Bulge being triaxial rather than oblate, with the near side at $\ell>0^{\circ}$. If this is indeed the case, then the observed surface brightness distribution no longer determines the three-dimensional shape uniquely (Stark 1977).

If we assume that the surfaces of constant volume emissivity are approximately ellipsoidal with semi-axes $a, b$ and $c$, then in order to determine the intrinsic shape of the Bulge, we must derive its luminosity profile, the scale-length $a$, the two axis ratios $b / a$ and $c / a$, and also the orientation of the ellipsoid. If the Galactic plane is one of the symmetry planes of the triaxial Bulge, then its orientation is fixed by the angle $\phi$ between the major axis of the Bulge and the line-of-sight from the Sun to the Galactic Center. In our convention $c / a$ is the axis ratio perpendicular to the Galactic plane, and $b / a$ is the axis ratio in the plane. Both axis ratios, and also $\phi$, may depend on radius. The deprojection of the observed surface brightness is not unique because a change in $b / a$ can be compensated by a change in $\phi$ so as to give the same projected distribution. It is therefore not surprising that the analysis by Blitz \& Spergel (1991b) does not give an accurate value for $\phi$, and that it does not provide a significant constraint on $b / a$.

While the non-uniqueness of the deprojection of the Bulge light is unavoidable, progress can be made by using measurements of higher quality. Combination of 
observations at different wavelengths should allow one to derive an accurate extinction correction, and to improve the estimate of the foreground contamination. The COBE measurements will be of great help here, as will the various large-scale surveys with infrared arrays that are being carried out (Glass 1993). Analysis of this data should provide useful constraints on the intrinsic shape of the Bulge, and should also shed light on the suggestion that the Bulge may be tipped relative to the Galactic plane (Blitz \& Spergel 1991b; Spergel 1992).

\subsection{STARCOUNTS}

Much of the classical work on individual stars in the Bulge has been restricted to a small number of special windows that are not heavily obscured by interstellar extinction and hence gives only limited information on the shape and structure of the entire Bulge (Frogel 1988). IRAS improved this situation dramatically. Habing et al. (1985) showed that a simple criterion based on the observed flux densities at 12 and 25 micron allows one to select AGB stars from the IRAS point source catalog. The distribution of these sources on the sky clearly shows the disk of the Galaxy, and the flattened Bulge (Habing 1988). After correction for the effects of confusion near the Galactic plane, the derived luminosity profile and axis ratio of the Bulge are consistent with the integrated light measurements (Harmon \& Gilmore 1988).

The recent interest in triaxiality has spurred re-analysis of the properties of various populations of Bulge stars. Nakada et al. (1991) investigated the luminosity function of a subsample of the IRAS AGB stars in the Bulge, and found that the stars at $\ell>0^{\circ}$ are brighter on average - and hence nearer to us - than those at $\ell^{\prime}<0^{\circ}$, in agreement with an earlier suggestion by Harmon \& Gilmore (1988).

Weinberg (1992a) re-analyzed the IRAS point source catalog, considered only objects with galactic latitude $|b|<3^{\circ}$, and used selection criteria which differ slightly from those of Habing et al. (1985). He assumed that all these stars have an absolute bolometric luminosity of $8000 L_{\odot}$, calculated individual bolometric corrections based on the observed IRAS colors, and assumed uniform extinction throughout the Galaxy. This gives distances for all the objects. The resulting galactic distribution appears to be lopsided. Weinberg argued that this is due to the apparent luminosity cutoff of the sample, which causes the exclusion of nearly all stars beyond $10 \mathrm{kpc}$. Analysis of a deeper, but incomplete, sample showed a more symmetric bar-like distortion, again with the near side at $\ell>0^{\circ}$. Instead of fitting a specific model to the distribution of stars, Weinberg calculated the coefficients of the harmonic expansion that fits the data best. He reconstructed a smooth density distribution from his expansion coefficients, ignoring the asymmetric terms. This yields a symmetric bar, out to about $5 \mathrm{kpc}$, with the near side at $\ell>0^{\circ}$, an axis ratio $b / a \sim 0.6$, and an orientation $\phi=36 \pm 10^{\circ}$. Varying the prescriptions for the bolometric correction and for the extinction, and allowing for a realistic spread in the intrinsic luminosities of the stars, did not change the main result-the existence of a bar-but influenced its properties. It remains to be seen whether the spatial extent of $5 \mathrm{kpc}$ found by Weinberg can be reconciled with the smaller size inferred from studies of the gas kinematics (§3.1). It is also not clear how the derived properties are influenced by confusion of sources near the Galactic plane. 
Weinberg (1992b) compared the counts of IRAS AGB stars at $|b|>3^{\circ}$ with simulated samples, and found that the central bulge seen in the distribution on the sky could be an artefact of extinction variations combined with the limited sensitivity of IRAS. Whereas in the Galactic plane IRAS barely detects the AGB stars at the center, at increasing $|b|$ the extinction decreases, and such stars can be detected along ever longer lines-of-sight, so that the counts are systematically larger. This effect is strongest towards the center of the Galaxy. The result is an apparent bulge in the distribution of stars in the IRAS map of the Galaxy.

The measurements of the integrated light ( $\$ 2.1)$, and notably the COBE data (Hauser et al. 1990), show clear evidence for a Bulge in the center of the Galaxy, in agreement with observations of nearby galaxies. The result of the experiments reported by Weinberg (1992b) do not indicate that the Galaxy contains no Bulge, but demonstrate that extinction is important even at 12 and 25 micron, and, more importantly, that results based on the IRAS database can be biased strongly by its limited sensitivity. These effects should not influence the detected asymmetry between the counts at positive and negative $\ell$.

A much improved analysis of the shape of the Bulge based on starcounts will be possible in the near future. The 2MASS (Kleinmann 1992) and DEIIS (Epchtein, Guglielmo, \& Burton 1992) sky surveys near 2 micron are expected to detect every AGB star in the Galaxy, and will provide ideal datasets for application of Weinberg's expansion method. Measurements in three bands will allow accurate correction for extinction, and the large number of sources will make a three-dimensional study feasible which takes into account a realistic luminosity function for the AGB stars. This should then also shed light on the question whether the structure detected by Weinberg (1992a) in his two-dimensional analysis is an elliptic thick disk, as suggested by Spergel (1992), or a cross-section through a triaxial Bulge.

Whitelock \& Catchpole (1992) investigated a sample of 104 Mira variables for which individual distances are known from the observed periods (Whitelock, Feast \& Catchpole 1991). The objects are located in two strips parallel to the Galactic plane with $-15<\ell<15^{\circ}$ and $7<|b|<8^{\circ}$. The Miras at $\ell>0^{\circ}$ have distance moduli that are $\sim 0.4$ magnitudes smaller on average than those at $\ell<0^{\circ}$. There is no difference between the distribution of distances within the two strips. A prolate bulge with $b / a=c / a \sim 0.25$ and $\phi \sim 45^{\circ}$ fits the data, in rough agreement with the results mentioned above. This work also shows that the elongated component extends to at least $1 \mathrm{kpc}$ above the Galactic plane. The Whitelock \& Catchpole study is a major step forward, because it is based on accurate individual, rather than statistical, distances. It is important to extend this work to smaller $|b|$. This is difficult-not only because of the increased extinction but also because many observations per star are required to determine the period-but is worth the effort.

Finally, we note that the RR Lyrae stars with known distances also show marginal evidence for a triaxial distribution, but with its near end at $\ell<0^{\circ}$ (Wesselink 1987; Le Poole \& Habing 1990). It will be interesting to see whether this result holds up when a larger sample is studied, since the RR Lyraes are old (Lee 1992), and-unlike the Miras and the AGB stars-presumably belong to the metalpoor halo. This may itself be triaxial, and indeed elongated in a direction opposite to the Bulge (Blitz \& Spergel 1991a; but see Kuijken \& Tremaine 1991, 1993). 


\section{Kinematics}

\subsection{GAS MOTIONS}

It has been known for a long time that the inner rotation curve of the Galaxy displays a prominent hump (Combes 1991; Liszt 1992). A natural explanation is to assume that the central part of the Galaxy is not axisymmetric, but contains a bar (de Vaucouleurs 1964) or a triaxial bulge. The simple closed orbits available to the gas are then elongated, and the gas velocity varies along the orbit. When viewed from the proper direction, i.e., by proper orientation of the Bulge, one may observe gas velocities that are larger than the circular velocity, and hence see a hump in the rotation curve. Liszt \& Burton (1980) gave an early description of the observations in terms of a simple kinematic model which is equivalent to motion on elliptic orbits (Kent 1992). Gerhard \& Vietri (1986) showed that the inner rotation curve can be reproduced by closed orbits in a prolate bulge with a stationary figure, a realistic density profile, and seen nearly broadside on. Burton \& Liszt (1993) have shown recently that the HI measurements are contaminated by absorption against the continuum radiation from the Galactic core. They correct for this effect by using observations of $\mathrm{OH}$ and $\mathrm{H}_{2} \mathrm{CO}$, and again confirm the presence of strong noncircular motions. The resulting azimuthally averaged velocity field gives a circular velocity curve that is consistent with the 2 micron light profile ( $\$ 2.1)$ and a constant mass-to-light ratio $\mathcal{M} / L$.

Binney et al. (1991) have constructed the most comprehensive model to date of the motions of both the atomic and the molecular gas in the inner kpc of the Galaxy. These authors assume the gas moves on non-selfintersecting closed orbits, and show that a rapidly rotating inner bar with $b / a=0.75$, a figure rotation rate of $63 \mathrm{~km} / \mathrm{s} / \mathrm{kpc}$ - so that the corotation radius lies at $2.4 \pm 0.5 \mathrm{kpc}$-and seen nearly end-on at $\phi=16^{\circ} \pm 2^{\circ}$, provides a natural explanation for the observed kinematics of the gas. The derived density profile resembles closely the one adopted by Kent (1992): the logarithmic slope is -1.75 in the inner Bulge, and approaches -3.5 at large radii. Designating the elongated central component as a bar or as a triaxial bulge may be a matter of semantics: the gas motions in the Galactic plane do not constrain the density distribution of the bar outside the plane, and, as Binney et al. point out, it is possible that the rapidly rotating bar is identical to the triaxial box-shaped Bulge evident in the integrated light and the star counts. The bar and the Bulge may also be separate Galactic components (\$4).

Proper modeling of the gas kinematics requires a careful hydrodynamical treatment, because the closed orbit approximation breaks down when the orbits become very elongated, or when the gas switches from one orbit family to another. Early hydrodynamical studies that discuss the effects of a triaxial bulge on the Galactic HI kinematics include van Albada (1985), Yuan (1984), and Mulder \& Liem (1986). The former author uses a bar which is similar to the one found by Binney et al. (1991), but the latter authors require a bar which has its nearest side at $\ell<0^{\circ}$ rather than at $\ell>0^{\circ}$. These studies use a rather crude approximation to the Galactic potential. Recent work by Jenkins \& Binney (1993) demonstrates that it is worthwhile to redo this kind of study, with a more up-to-date potential and state-of-the-art hydrodynamical schemes. 


\subsection{STELLAR RADIAL VELOCITIES}

Much of the classical spectroscopy of Bulge stars was restricted to Baade's Window. At present, radial velocities are available for a variety of populations in a number of windows. These include $\mathrm{K}$ and $\mathrm{M}$ giants, carbon stars, planetary nebulae and RR Lyrae stars. Kent (1992) summarizes many of the measurements, including those of the integrated light. More recent studies include work on $\mathrm{K}$ giants in fields between 1.5 and $2 \mathrm{kpc}$ from the center (Minniti et al. 1992; Harding \& Morrison 1993).

This body of work shows that the Bulge rotates, and has a mean line-of-sight velocity $\left\langle v_{\text {los }}\right\rangle$ of $5-10 \mathrm{~km} / \mathrm{s} /{ }^{\circ}$, so that $\left\langle v_{\text {los }}\right\rangle$ may reach about $80 \mathrm{~km} / \mathrm{s}$ at $1 \mathrm{kpc}$ along the major axis. Different gradients of $\left\langle v_{\text {los }}\right\rangle$ found for different samples are sometimes taken as evidence for a dependence of the kinematics on other properties of the stellar population, such as abundance or age. However, such differences may also be caused by the customary but highly suspect fitting of linear regression lines to samples that often have different radial extent. There is no a priori reason to expect a linear dependence of $\left\langle v_{\mathrm{los}}\right\rangle$ on radius ( $\left.\$ 4\right)$.

The line-of-sight radial velocity dispersion $\sigma_{\text {los }}$ in the Bulge increases from $\sim 80$ $\mathrm{km} / \mathrm{s}$ at $10 \mathrm{pc}$ to $\sim 115 \mathrm{~km} / \mathrm{s}$ at a few hundred pc from the center. At larger distances $\sigma_{\text {los }}$ decreases again, in a manner which is consistent with the inward increase of $\sigma_{\text {los }}$ measured in the old disk (Lewis \& Freeman 1989; Carney, Latham \& Laird 1990). There is evidence that $\sigma_{\text {los }}$ increases in the inner 3 pc (Sellgren et al. 1990), but not all authors agree (Rieke \& Rieke 1988). Such an increase could be caused by a central black hole or nuclear star cluster with a mass of a few times $10^{6} M_{\odot}$.

In order to investigate the stellar kinematics of the entire Bulge, tracers are needed that can be detected at radio wavelengths, such as OH/IR stars and planetary nebulae. The OH/IR stars are most useful: these AGB objects are surrounded by an expanding dust shell, which can be readily identified by means of its $\mathrm{OH}$ emission at $18 \mathrm{~cm}$ (Fig. 2). The spherical geometry of the shell causes the line profiles to be double-peaked, so the measured velocity width is twice the expansion velocity $v_{\text {exp }}$ of the shell, and the mean velocity is the radial velocity $v_{\text {los }}$ of the embedded star. Because radio measurements are not hampered by Galactic obscuration, $v_{\text {los }}$ can be determined for $\mathrm{OH} / \mathrm{IR}$ stars throughout the Galaxy. By contrast, radial velocities of planetary nebulae are generally based on optical spectroscopy, so that foreground extinction remains a problem (Stasińska 1993).

Early radio surveys of the central few hundred pc (Habing et al. 1983; Winnberg et al. 1985) found a few dozen OH/IR stars, and showed that the inner Bulge may well rotate rapidly, with $\left\langle v_{\text {los }}\right\rangle \sim 100 \mathrm{~km} / \mathrm{s}$ at less than $100 \mathrm{pc}$ from the center, and with $\sigma_{\text {los }}$ between 60 and $140 \mathrm{~km} / \mathrm{s}$. Lindqvist et al. $(1992 \mathrm{a}, \mathrm{b})$ have recently completed a large VLA survey which produced $134 \mathrm{OH} / \mathrm{IR}$ stars in the central 100 pc. Their kinematic properties seem to depend on the physical properties of the stars: those with $v_{\text {exp }}<18 \mathrm{~km} / \mathrm{s}$, which are thought to be low mass old objects, have $\sigma_{\text {los }}=82 \pm 7 \mathrm{~km} / \mathrm{s}$, while stars with $v_{\exp }>18 \mathrm{~km} / \mathrm{s}$, which are thought to be more massive, and younger, have $\sigma_{\text {los }}=65 \pm 6 \mathrm{~km} / \mathrm{s}$. The fast rotation found earlier is confirmed: $\left\langle v_{\text {los }}\right\rangle$ reaches over $100 \mathrm{~km} / \mathrm{s}$ at $\sim 40 \mathrm{pc}$ from the center. This is comparable to the circular velocity, and suggests that at least part of this OH/IR star population may be in a rotationally supported disk. 


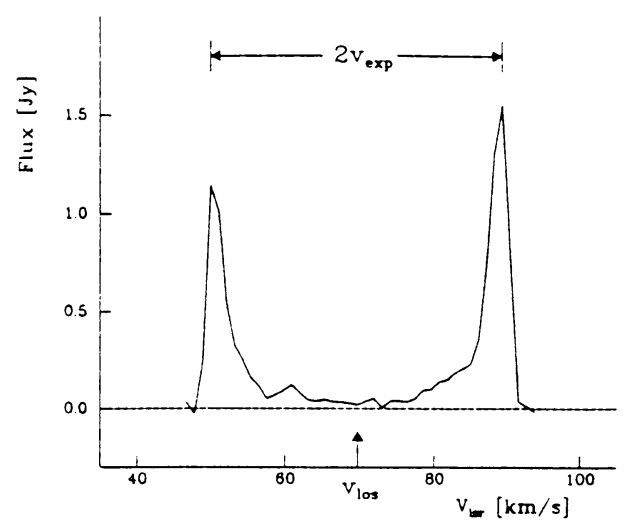

Fig. 2. Line profile of an $\mathrm{OH} / \mathrm{IR}$ star at $18 \mathrm{~cm}$. The velocity difference between the two peaks is twice the expansion velocity of the circumstellar dust shell, and the mean velocity of the peaks equals the radial velocity of the central star. The data are for the source OH359.954-0.041 (van Langevelde 1992).

A number of surveys of IRAS AGB stars have been carried out at $18 \mathrm{~cm}$, resulting in radial velocities for several hundred OH/IR stars in the Bulge (te Lintel-Hekkert et al. 1989, 1991). This sample has $\sigma_{\text {los }} \sim 110 \mathrm{~km} / \mathrm{s}$ and $\left\langle v_{\text {los }}\right\rangle \sim 10 \mathrm{~km} / \mathrm{s} /{ }^{\circ}$ (Dejonghe 1993b), in good agreement with other measurements, notably those of Mira variables (Menzies 1990) and planetary nebulae (Kinman, Feast \& Lasker 1988; Acker et al. 1991), which are in evolutionary stages bracketing the OH/IR stage. Radial velocities based on the $\mathrm{SiO}$ maser emission of $\mathrm{OH} / \mathrm{IR}$ stars with dense dust shells also give similar values for $\left\langle v_{\text {los }}\right\rangle$ and $\sigma_{\text {los }}$ (Nakada et al. 1993).

It is unfortunate that the IRAS selected OH/IR sample is incomplete near the Galactic plane, due to the confusion limitations of the IRAS point source catalog. As a result, there is hardly any overlap with the fast-rotating Lindqvist et al. (1992a) sample near the Galactic Center (see Fig. 1 of Dejonghe 1993b), so that the possible presence of an extended disk component in the Bulge remains uncertain. The systematic, sensitive, and unbiased radio survey of the entire Bulge at 18 $\mathrm{cm}$, which is being carried out by Habing and his collaborators, will improve this situation considerably.

The distribution of $v_{\text {los }}-\left\langle v_{\text {los }}\right\rangle$ for the known OH/IR stars in the inner Bulge appears to be nearly Gaussian, but it should be realized that surveys for these stars are often done in a limited velocity range, due to the finite bandwidth of receivers. Van Langevelde et al. (1992b) sampled a large velocity interval, and showed that in addition to Baud's star, two more OH/IR stars close to the center have $v_{\text {los }} \sim 350$ $\mathrm{km} / \mathrm{s}$. Since deviations from a Gaussian velocity distribution are expected (\$4), especially if the Bulge is triaxial, it is important to search for OH/IR stars in a large velocity interval, even if the bulk of the objects lies in a more limited range. By the same argument, "obvious outliers" in samples of stars with optical radial velocities should be deleted with caution. 


\subsection{PROPER MOTIONS}

In a very exciting recent development, it is now possible to measure proper motions of Bulge stars, which are of the order of $3 \mathrm{marcsec} / \mathrm{yr}$. This provides information on the two components of the stellar velocity vectors that so far could not be measured directly. An example is the study of $\mathrm{K}$ and $\mathbf{M}$ giants in Baade's Window by Spaenhauer, Jones \& Whitford (1992). Assuming a distance to the Galactic Center of $7.7 \mathrm{kpc}$, they find $\sigma_{\ell}=115 \pm 4 \mathrm{~km} / \mathrm{s}$ and $\sigma_{b}=100 \pm 4 \mathrm{~km} / \mathrm{s}$ in this minor axis field; these values may depend somewhat on the metallicity of the stars.

Proper motions of OH/IR stars in the Bulge can be measured by VLBI techniques in the near future. In the inner $100 \mathrm{pc}$ this cannot be done at $18 \mathrm{~cm}$, because interstellar scattering limits the resolution (van Langevelde et al. 1992a). However, since the scattering scales with $\lambda^{2}$, use of the $\mathrm{H}_{2} \mathrm{O}(\lambda 1.3 \mathrm{~cm})$ or $\mathrm{SiO}(\lambda 0.7 \mathrm{~cm})$ maser emission of these objects will allow accuracies of $\sim 10 \mathrm{~km} / \mathrm{s}$ in $5-10 \mathrm{yr}$.

\section{Dynamics}

Can we reconcile the morphology of the Bulge with the kinematics? We have seen in $§ \S 2.1,2.2$ and 3.1 that the integrated light, the starcounts, and the kinematics of the gas all seem to point to an elongated Bulge, and that the various studies generally agree on its properties, even though differences in detail remain. What about the stellar motions? This is a difficult question to answer, because many different intrinsic velocity distributions may be consistent with the same mass model, so that it is not easy to constrain the shape, the density profile and the entire intrinsic velocity distribution in the Bulge all at once just from the measured stellar kinematics. For this reason the usual approach is to first specify a potential and a mass distribution, and then to calculate the observed kinematics of the stellar component for various velocity distribution functions $f$. The result of this dynamical modeling is then compared to the observations. If no model velocity distribution can be found that fits the data, the potential and/or mass model was chosen incorrectly. If there are solutions, then this does not prove that the model is correct, but only shows that it is at least consistent with the available data, and allows a certain range of possible distribution functions. Whether the inferred distribution functions are in fact plausible is then a matter for theories of bulge formation.

To date, dynamical modeling of the Bulge has been restricted to spherical or axisymmetric geometries, and has made use of the Jeans equations, i.e., considered only the first and second moments of the velocity distribution function $f$. The most comprehensive model that is available is due to Kent (1992). His mass model for the Bulge fits the 2.4 micron integrated light distribution (Fig. 1) for a constant $\mathcal{M} / L$. The associated rotation curve agrees with the observed gas kinematics (§3.1). Kent assumes that the distribution function $f$ is of the special form $f=f\left(E, L_{z}\right)$, where $E$ is the orbital energy, and $L_{z}$ is the component of angular momentum around the short axis of the Bulge, taken as the $z$-axis. This guarantees that the second velocity moments $\left\langle v_{R}^{2}\right\rangle$ and $\left\langle v_{z}^{2}\right\rangle$ in the $R$ and $z$ directions, respectively, are equal everywhere. They follow from the Jeans equations, together with the remaining second moment $\left\langle v_{\phi}^{2}\right\rangle$. By symmetry, there can be no mean streaming in the $R$ and 


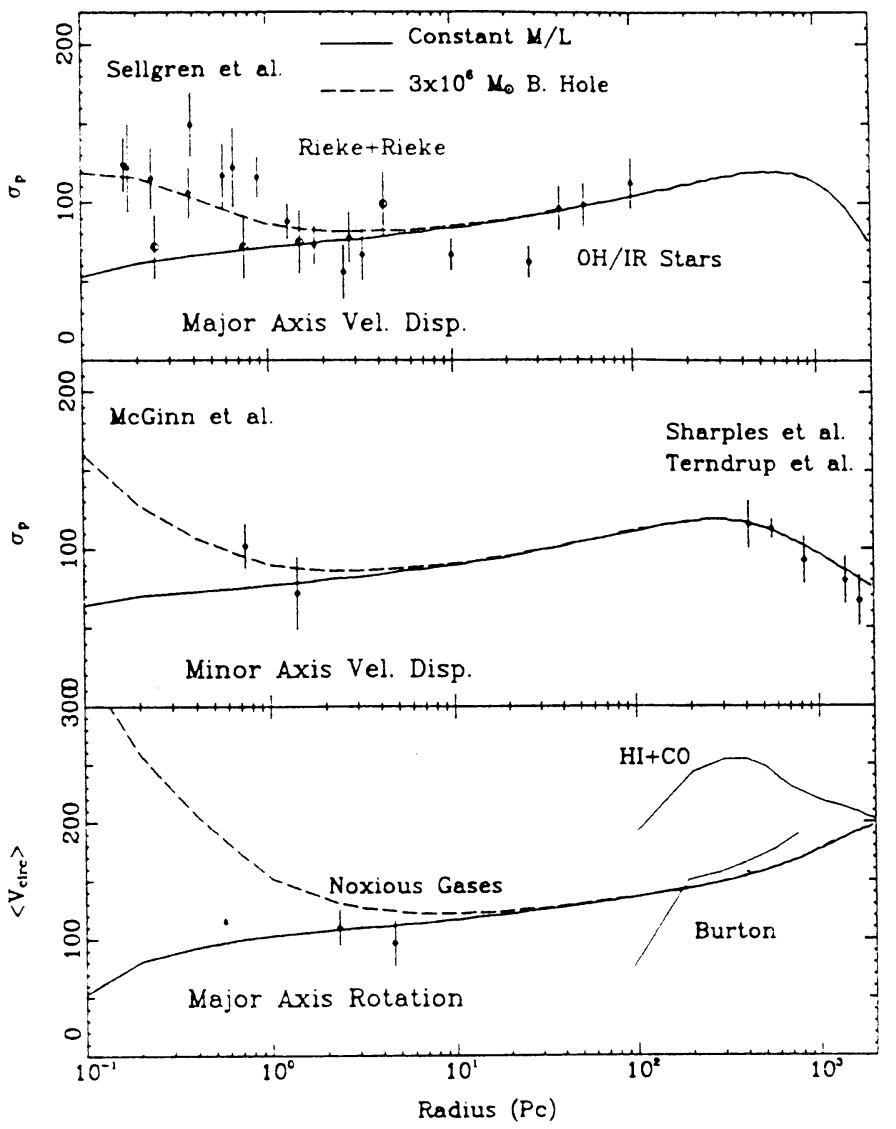

Fig. 3. Comparison of Kent's oblate Bulge model with kinematic data. Top \& middle panels give $\sigma_{\text {los }}$ along the major and the minor axis, respectively. Bottom panel gives $v_{c}$ along the major axis. Solid lines are for constant $\mathcal{M} / L$, and dashed lines indicate a model with a central black hole of $3 \times 10^{6} M_{\odot}$. See text and Kent (1992) for more details.

$z$ directions, so the velocity dispersions $\sigma_{R}$ and $\sigma_{z}$ are equal everywhere. Kent now chooses the mean streaming velocity in the $\phi$-direction in such a way that $\left\langle v_{\phi}^{2}\right\rangle-\left\langle v_{\phi}\right\rangle^{2}=\left\langle v_{R}^{2}\right\rangle=\left\langle v_{z}^{2}\right\rangle$. This makes all three velocity dispersions equal at every point. Such a model is often referred to as an oblate isotropic rotator.

Figure 3 shows $\sigma_{\text {los }}$ along the major and the minor axis of Kent's model, as well as the circular velocity $v_{c}$ as function of radius. The solid lines are for a model with constant $\mathcal{M} / L$. The curves rise proportional to $r^{0.075}$ in the inner Bulge, as expected for a density profile that decreases as $r^{-1.85}$. Specifically, this means an increase by a factor of 1.4 in $\sigma_{\mathrm{los}}$ between 5 and $500 \mathrm{pc}$. Beyond the knee in the density profile (Fig. 1) $\sigma_{\text {los }}$ decreases, as expected, while $v_{c}$ continues to increase until it reaches $\sim 200 \mathrm{~km} / \mathrm{s}$ at $2 \mathrm{kpc}$ from the center. The dashed lines are the expected curves when a central black hole of $3 \times 10^{6} M_{\odot}$ is included. The data 
TABLE I

Comparison of Kent's oblate Bulge model with recent kinematic observations.

\begin{tabular}{|c|c|c|c|c|c|c|}
\hline $\begin{array}{l}\ell \\
\circ\end{array}$ & $\begin{array}{l}b \\
0\end{array}$ & $\begin{array}{l}\text { Observed } \\
\sigma_{\text {los }} \\
\mathbf{k m} / \mathbf{s}\end{array}$ & $\begin{array}{l}\left\langle v_{\text {los }}\right\rangle \\
\mathrm{km} / \mathrm{s}\end{array}$ & $\begin{array}{l}\text { Model } \\
\sigma_{\text {os }} \\
\mathbf{k m} / \mathbf{s}\end{array}$ & $\begin{array}{l}\left\langle v_{\text {los }}\right\rangle \\
\mathrm{km} / \mathrm{s}\end{array}$ & Source \\
\hline 8 & 7 & $85 \pm 7$ & $45 \pm 10$ & 91 & 42 & Minniti et al. (1992) \\
\hline 12 & 3 & $68 \pm 6$ & $77 \pm 9$ & 65 & 90 & Minniti et al. (1992) \\
\hline-10 & -10 & $67 \pm 6$ & $82 \pm 8$ & 77 & 47 & Harding \& Morrison (1993) \\
\hline
\end{tabular}

points are from Kent's compilation of recent observations, and are well-fit by the model. The value of $\mathcal{M} / L$ has been set by requiring that the model reproduces the observed velocity dispersion in Baade's Window. This gives $\mathcal{M} / L_{K}=1.0 \pm 0.15$ in solar units.

Any oblate model with $f=f\left(E, L_{z}\right)$ predicts $\sigma_{b}=\sigma_{\text {los }}$ along the minor axis, as a consequence of the fact that $\sigma_{R}=\sigma_{z}$ everywhere. This can be tested for Kent's model by comparing it with the proper motions in Baade's Window. For the distance to the Galactic Center of $8 \mathrm{kpc}$ favored by Kent, the Spaenhauer, Jones $\&$ Whitford (1992) measurements are: $\left(\sigma_{\ell}, \sigma_{b}\right)=(119 \pm 4,104 \pm 4) \mathrm{km} / \mathrm{s}$, while $\sigma_{\text {los }}=113 \pm 6 \mathrm{~km} / \mathrm{s}$ (Mould 1983; Sharples, Walker \& Cropper 1990; Rich 1990; Tyson \& Rich 1991). Kent (priv. comm.) predicts $\left(\sigma_{\ell}, \sigma_{b}, \sigma_{\text {los }}\right)=(120,113,113)$ $\mathrm{km} / \mathrm{s}$ for his model, which agrees remarkably well with the observations. A further test of the model is provided by the radial velocities obtained by Minniti et al. (1992) and by Harding \& Morrison (1993). Kent kindly made available his unpublished predictions for these fields. They are compared with the observations in Table 1. The agreement is excellent, except for $\left\langle v_{\text {los }}\right\rangle$ in the Harding \& Morrison field. It is not clear whether this is caused by an inaccurate foreground correction, has to do with the definition of the sample, or is a first hint that the Bulge is not an oblate isotropic rotator.

Kent's model is the simplest oblate Bulge model that can be constructed, and yet it fits essentially all the available data. This is quite remarkable, not only because many other velocity distributions are possible in principle, but also in view of the strong indications that the Bulge is not oblate but triaxial ( $\$ 2$ and 3 ).

At the same time, the observed stellar kinematics do constrain the shape, the density profile, and the velocity distribution of the Bulge: not every model fits the available data. To illustrate this, we consider a simple scale-free model for the inner Bulge with a potential $\psi \propto 1 /\left(R^{2}+z^{2} / q^{2}\right)^{\alpha / 2}$, where $q$ and $\alpha$ are constants, and we ignore the putative central black hole. The associated density profile is a power law with logarithmic slope $-(2+\alpha)$. The even part of the self-consistent distribution function $f\left(E, L_{z}\right)$ of such models is given by $f\left(E, L_{z}\right)=A L_{z}^{2} E^{4 / \alpha-3 / 2}+C E^{2 / \alpha-1 / 2}$, with $A$ and $C$ constants (Evans 1993b). The limiting case $\alpha \rightarrow 0$ has a logarithmic potential and is discussed by Toomre (1982) and Evans (1993a). We note that none 


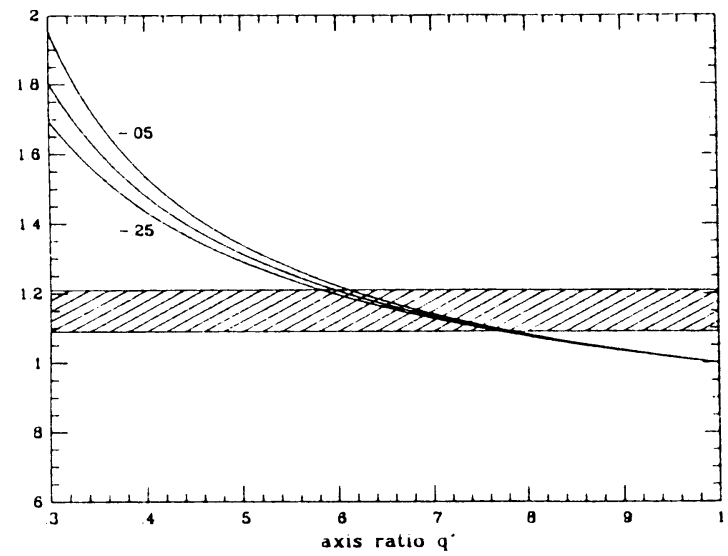

Fig. 4. Relation between $\sigma_{\ell} / \sigma_{b}$ on the minor axis and the observed axis ratio $q^{\prime}$ of the simple power law models for the inner Bulge, seen edge-on. The curves correspond to density profiles $\rho \propto 1 / r^{2+\alpha}$, with $\alpha=-0.05,-0.15$, and -0.25 . The hatched area indicates the range of $\sigma_{\ell} / \sigma_{b}$ compatible with the measurements in Baade's Window.

of these models have Gaussian velocity distributions, because $f$ is not a simple exponential of the energy. The value of $q$ determines the flattening of the models; they become increasingly peanut-shaped with decreasing $q$. For all these models the observed second moments of the line-of-sight velocities and the proper motions can be given explicitly at any point. The same is true for the first moments of the oblate isotropic rotator along the principal axes. The full expressions will be given elsewhere. Here we limit ourselves to one example, and show in Figure 4 the observed value of $\sigma_{\ell} / \sigma_{b}$ along the minor axis of these models as function of the observed flattening $q^{\prime}$, for various values of $\alpha$. For edge-on observation the relation between $\sigma_{\ell} / \sigma_{b}$ and $q^{\prime}$ is given by:

$$
\left(\frac{\sigma_{\ell}}{\sigma_{b}}\right)^{2}=\frac{q^{2}+1-2 \alpha}{3 q^{2}-1-2 \alpha}, \quad q^{\prime}=q\left(\frac{q^{2}-\alpha}{1-\alpha q^{2}}\right)^{1 /(1+\alpha)} .
$$

We conclude that the value $\alpha=-0.15$ used by Kent (1992), or the value $\alpha=$ -0.25 advocated by Binney et al. (1991), can only be reconciled with $\sigma_{\ell} / \sigma_{b}=$ $1.15 \pm 0.06$ in Baade's Window for $q^{\prime}$ between 0.6 and 0.75 , in good agreement with the observations $(\$ 2.1)$. This by no means proves that the Bulge is oblate with $f=f\left(E, L_{z}\right)$, but illustrates that meaningful modeling of the intrinsic velocity distribution requires accurate knowledge of the shape of the Bulge. The figure furthermore shows that using simple models with a $1 / r^{2}$ density profile is adequate for some purposes, but not when they are restricted to be spherical.

Kent's model is similar in spirit to the oblate models for elliptical galaxies constructed by Binney, Davies \& Illingworth (1990) and van der Marel (1991). These authors restrict themselves to $f=f\left(E, L_{z}\right)$ but, following Satoh (1980), they consider a variety of mean streaming motions by taking $\left\langle v_{\phi}\right\rangle^{2}=k^{2}\left(\left\langle v_{\phi}^{2}\right\rangle-\left\langle v_{R}^{2}\right\rangle\right)$, with $k$ a constant. The oblate isotropic rotator has $k=1$. In the inner Bulge, the 
value of $k$ is likely to be near one: the simple power law model with $\alpha=-0.15$, $q^{\prime} \sim 0.65$ and $k=1$ gives $\left\langle v_{\text {los }}\right\rangle / v_{c} \sim 0.35$ along the major axis, in agreement with the few available observations and with Kent's statement that $\left\langle v_{\text {los }}\right\rangle \sim 40 \mathrm{~km} / \mathrm{s}$ throughout the inner part of his model (Fig. 3).

Instead of working with the first and second moments of the observed velocity distribution, one would like to compare this distribution directly with the model prediction. This is easy for the power-law models (Evans 1993b). To calculate $f\left(E, L_{z}\right)$ for more realistic models it may be possible to employ the method by Hunter \& Qian (1993), who made a major breakthrough in the long-standing problem of the practical calculation of $f\left(E, L_{z}\right)$ for axisymmetric systems. Their work transforms the calculation of $f\left(E, L_{z}\right)$ from an often frustrating chase through tables of integral transforms and special functions, to the straightforward (numerical) evaluation of $f$ as a well-defined contour integral. It will be interesting to see whether this can be used to obtain $f$ for a model like the one proposed by Kent.

One disadvantage of using velocities of individual stars to study the dynamics of the Bulge is the need for many measurements before a velocity distribution can be determined-or even just its first and second moments. For this reason it is valuable to redo and extend the spectroscopic measurements of the integrated light (Freeman et al. 1988), preferably in the calcium triplet region, or in the infrared (McGinn et al. 1989). Various methods now exist to extract not just $\left\langle v_{\text {los }}\right\rangle$ and $\sigma_{\text {los }}$ but also the entire line-of-sight velocity distribution from such data (e.g., Rix \& White 1992; van der Marel \& Franx 1993).

Before considering truly anisotropic models in which $\left\langle v_{R}^{2}\right\rangle \neq\left\langle v_{z}^{2}\right\rangle$ and $\left\langle v_{R} v_{z}\right\rangle \neq$ 0 , it is useful to investigate simple models with an embedded disk. This can be done following the approach of Evans \& Collett (1993), who give two-integral distribution functions for exponential disks in a logarithmic halo potential, or by using the Jeans equations (Cinzano \& van der Marel 1993). The resulting models will help to elucidate the properties of the population of fast-rotating OH/IR stars in the inner $100 \mathrm{pc}$ (Lindqvist et al. 1992b). A general method to test whether an edge-on oblate model has $f=f\left(E, L_{z}\right)$ is described by Merrifield (1991). Its application will require further kinematic observations, however.

Anisotropic oblate models have $f=f\left(E, L_{z}, I_{3}\right)$ where $I_{3}$ is a(n approximate) third integral of motion. Selfconsistent calculation of such $f$ 's is sometimes possible semi-analytically (Binney \& Petrou 1985), but generally requires numerical techniques such as linear programming (Schwarzschild 1979; Richstone 1980, 1984; Levison \& Richstone 1985a, b; Fillmore \& Levison 1989). More direct methods are available for the solution of the Jeans equations when $\left\langle v_{R}^{2}\right\rangle \neq\left\langle v_{z}^{2}\right\rangle$ and $\left\langle v_{R} v_{z}\right\rangle \neq 0$ (Bacon 1985; Fillmore 1986), and these should be applied to the Bulge. Kent's (1992) simple estimate based on spherical models already shows that the observations in Baade's Window will provide strong constraints on the allowed anisotropy of the velocity distribution in the Bulge.

Te Lintel-Hekkert, Dejonghe \& Habing (1991) have taken a slightly different approach. They choose a potential, define certain smooth components with simple distribution functions $f$, calculate the observed properties for each of these separately, and then use a quadratic programming method (Dejonghe 1989) to determine which combination, if any, reproduces the observations. Application to the 
OH/IR stars in the Bulge (excluding the Lindqvist et al. 1992a sample) again shows that $\rightarrow$ so far- $f\left(E, L_{z}\right)$ components in an oblate potential are consistent with the observations (Dejonghe 1993b). This approach has considerable promise. The effects of an anisotropic velocity distribution and of a triaxial shape can be incorporated by considering models with separable potentials (cf. Dejonghe \& Laurent 1991). Unfortunately, self-consistent models of this kind of necessity have a finite density core rather than a central cusp such as observed in the Bulge, and also must have stationary figures. Separable models with cusps can be built non-consistently (Dejonghe 1993a), but so far there is little evidence that $\mathcal{M} / L$ varies in the Bulge, at least outside $3 \mathrm{pc}$. Furthermore, the triaxial Bulge may have a figure rotation rate of $63 \mathrm{~km} / \mathrm{s} / \mathrm{kpc}$ (Binney et al. 1991). Although stationary scale-free triaxial models of the kind constructed by Levison \& Richstone (1987) might be useful for the innermost part of the Bulge (but see Schwarzschild 1993), models for the entire Bulge must include non-zero figure rotation, a density profile which is not a power law, and must allow for the possibility of a separate flat bar in a triaxial Bulge. Construction of such models is a non-trivial project, which will require substantial numerical effort.

Dynamical modeling of the Bulge should be accompanied by careful N-body simulations, in order to investigate its evolution and interaction with other Galactic components (Hernquist \& Weinberg 1992). Studies of this kind will allow an exploration of various formation scenarios: they have shown already that an initially flat bar in a disk galaxy may grow fatter in time, become box-shaped, and either form a bulge, or a metal-rich component in a pre-existing bulge (Combes et al. 1990; Pfenniger \& Norman 1990; Raha et al. 1991; Pfenniger \& Friedli 1991). The effects of (the growth of) a central point mass can be investigated also.

The success of the oblate $f\left(E, L_{z}\right)$ models shows that it is not easy to detect the signature of the triaxiality of the Bulge in the available stellar kinematic data, and bence to constrain more sophisticated dynamical models and simulations. One of the first hints may be the observation of high velocity OH/IR stars near the Galactic Center. Van Langevelde et al. (1992b) show that the observed number is consistent with these stars being on the elongated orbits needed to support a triaxial Bulge, but only if the Bulge is seen nearly end-on, which is precisely the geometry favored by Binney et al. (1991). Various other tests for triaxiality have been proposed. These include searching for stars in the solar neighborhood that are on very elongated orbits which bring them close to the Galactic Center. The expected number of such stars is influenced by the shape of the Bulge (Spergel 1992). Along similar lines, the present distribution of orbital elements of the globular clusters may still contain evidence for enhanced destruction of such clusters in the past due to dynamical friction in a triaxial potential (Long, Ostriker \& Aguilar 1992). Another signature of triaxiality would be the detection of a gradient in $\left\langle v_{\mathrm{los}}\right\rangle$ along $\ell=0^{\circ}$. This could be due to the motion of stars on the long axis tube orbits expected in a triaxial Bulge, but could also be caused by a tipping of the Bulge with respect to the Galactic plane (Spergel 1992). There clearly is room for a lot more work on the dynamics of the Bulge. 


\section{Other Bulges}

Various lines of evidence suggest that spiral bulges as a class are not oblate. The position angles of the apparent major axis of the bulge and the disk of spiral galaxies often differ from each other. This is a natural consequence of triaxiality, and is caused by projection. A well-known example is the bulge of M31 (Stark 1977). Bertola, Vietri \& Zeilinger (1991) studied a sample of 32 bulges, and showed that if disks are round, then bulges as a class are indeed triaxial, and have shapes similar to elliptical galaxies. The derived distribution of shapes may be incorrect, however, as photometrically the disks of spirals are not round, but instead are slightly elongated, with an axis ratio close to 0.9 (Binney \& de Vaucouleurs 1981; Kuijken \& Tremaine 1991, 1993; Franx \& de Zeeuw 1992). Derivation of the intrinsic shapes of bulges will require inclusion of kinematic data, just as was done for elliptical galaxies (Binney 1985; Franx, Illingworth \& de Zeeuw 1991).

Individual bulges also show signs of triaxiality. The regular gas velocity field of NGC 4845 is well-fit by motion on elongated closed orbits in a triaxial bulge, with axis ratios $b / a=0.74 \pm 0.06$ and $c / a=0.60 \pm 0.06$ (Bertola, Rubin \& Zeilinger 1989; Gerhard, Vietri \& Kent 1989). Stellar absorption line measurements of bulges are consistent with rotationally supported axisymmetric models, when the disk potential is taken into account (Jarvis \& Freeman 1985; Rowley 1988; Kent 1989). The one known exception is the curious galaxy NGC 4550, which has two counter-rotating stellar disks, and a stationary bulge ( $\mathrm{Rix}$ et al. 1992). The data are consistent also with triaxial shapes with substantial internal streaming, and/or figure rotation. The various indications that the Galaxy contains a triaxial bulge, even though the stellar kinematics so far appears to be well-described by $f\left(E, L_{z}\right)$ axisymmetric models, are therefore fully in line with what we know about other bulges.

\section{Conclusions}

Observations of the integrated light, starcounts, and measurements of the kinematics of the atomic and molecular gas in the inner region of the Galaxy all indicate that the Galactic Bulge is triaxial, with its near side at positive longitude, and its long axis close to the line-of-sight to the Galactic Center. In the inner regions the density profile is a power law with logarithmic slope $-1.8 \pm 0.05$, which steepens to $-3.7 \pm 0.2$ beyond $\sim 400 \mathrm{pc}$ along the minor axis. The observed axis ratio in the direction perpendicular to the Galactic plane is $0.65 \pm 0.05$. The COBE observations, and the starcounts to be done with the DENIS and 2MASS surveys, will further delineate the shape and orientation of the Bulge. Studies of populations of variable stars for which accurate individual distances can be determined will also be very useful for this purpose. Improved modeling of the gas kinematics will require detailed hydrodynamical simulations, and should provide better constraints on the figure rotation and the elongation of the Bulge.

The consequences of triaxiality for the dynamics of the Bulge remain largely unexplored, for two reasons. First, radial velocities are available for a modest number of Bulge stars only, and are mostly restricted to certain windows. So far, the data are consistent with the simple oblate $f=f\left(E, L_{z}\right)$ model of Kent (1992), which is 
well-approximated by a simple power struction of anisotropic triaxial models with realistic density profiles and non-zero figure rotation is difficult and time-consuming.

This unsatisfactory situation should improve in the near future. New radial velocity surveys, such as the unbiased radio survey of OH/IR stars in the entire Bulge, and especially the work on proper motions, will provide a superior stellar kinematic dataset for the Bulge, even though it remains non-trivial to correct for foreground contamination by the disk. These observational programs should also clarify the nature of the sample of fast rotating OH/IR stars seen in the inner 100 pc, and in particular whether the Bulge contains an extended disk component.

On the theoretical front one should construct velocity distribution functions for the triaxial mass model that best fits the gas kinematics, the starcounts and the integrated light measurements, and then investigate which of these are preferred by the different populations of stars in the Bulge. This will require considerable numerical effort, but should help to constrain different formation scenarios for the Bulge, such as formation by direct gaseous infall from the halo, or by thickening of the disk. It is crucial to complement this dynamical modeling with careful $\mathbf{N}$-body simulations which incorporate a realistic disk, halo and Bulge. This will allow a study of the evolution of e.g., an initially flat bar into a triaxial Bulge. Comparison with the observed correlations between kinematics and abundances will result in a much improved understanding of the formation history of the Bulge.

\section{Acknowledgements}

It is a pleasure to acknowledge enlightening discussions with Marijn Franx and Huib-Jan van Langevelde, and especially with Wyn Evans and Steve Kent, who also generously gave permission to quote some of their unpublished results.

\section{References}

Acker, A., Köppen, J., Stenholm, B., \& Raytchev, B., 1991. A\&AS, 89, 237.

Bacon, R., 1985. A\&A, 143, 84.

Becklin, E.E., \& Neugebauer, G., 1968. ApJ, 151, 145.

Bertola, F., Rubin, V.C., \& Zeilinger, W.W., 1989. ApJ, 345, L29.

Bertola, F., Vietri, M., \& Zeilinger, W.W., 1991. ApJ, 374, L13.

Binney, J.J., 1985. MNRAS, $212,767$.

Binney, J.J., Davies, R.L., \& nlingworth, G.D., 1990. ApJ, 361, 78.

Binney, J.J., \& de Vaucouleurs, G., 1981. MNRAS, 194, 679.

Binney, J.J., Gerhard, O.E., Stark, A.A., Bally, J., \& Uchida, K.I., 1991. MNRAS, $252,210$.

Binney, J.J., \& Petrou, M., 1985. MNRAS, 214, 449.

Blitz, L., \& Spergel, D.N., 1991a. ApJ, 370, 205.

Blitz, L., \& Spergel, D.N., 1991b. ApJ, 379, 631.

Burton, W.B., \& Liszt, H.S., 1993. A\&A, in press.

Carney, B.W., Latham, D.W., \& Laird, J.B., 1990. AJ, 99, 572.

Cinzano, P.-A., \& van der Marel, R.P., 1993. In Structure, Dynamics, and Chemical Evolution of Early-Type Galaxies, eds I.J. Danziger, W.W. Zeilinger, \& K. Kjär (ESO Garching), p. 105.

Combes, F., 1991. ARAA, 29, 195.

Combes, F., Debbash, F., Friedli, D., \& Pfenniger, D., 1990. A\&A, 233, 82.

Dejonghe, H.B., 1989. ApJ, 343, 113.

Dejonghe, H.B., 1993a. In Structure, Dynamics, and Chemical Evolution of Early-Type Galaxies, eds I.J. Danziger, W.W. Zeilinger, \& K. Kjär (ESO/EIPC, Garching), p. 337. 
Dejonghe, H.B., 1993b. In IAU Symposium 155, Planetary Nebulae, eds R. Weinberger, A. Acker (Kluwer, Dordrecht), in press.

Dejonghe, H.B., \& Laurent, D., 1991. MNRAS, 252, 606.

de Vaucouleurs, G., 1964. In IAU Symposium 20, The Galaxy and the Magellanic Clouds, eds F.J. Kerr \& A.W. Rodgers (Sydney: Australian Academy of Science), p. 195.

Epchtein, N., Guglielmo, F., \& Burton, W.B., 1992. In IAU Symposium 149, The Stellar Populations of Galaxies, eds. B. Barbuy \& A. Renzini (Kluwer, Dordrecht), p. 414.

Evans, N.W., 1993a. MNRAS, 260, 191.

Evans, N.W., 1993b. In preparation.

Evans, N.W., \& Collett, J.L., 1993. MNRAS, in press.

Fillmore, J.A., 1986. AJ, $91,1096$.

Fillmore, J.A., \& Levison, H.F., 1989. AJ, 97, 57.

Franx, M., Illingworth, G.D., \& de Zeeuw, P.T., 1991. ApJ, 383, 112.

Franx, M., \& de Zeeuw, P.T., 1992. ApJL, 392, L47.

Freeman, K.C., 1987. ARAA, 25, 603.

Freeman, K.C., de Vaucouleurs, G., de Vaucouleurs, A., \& Wainscoat, R.J., 1988. ApJ, 325, 563.

Frogel, J.A., 1988. ARAA, 26, 51.

Gerhard, O.E., \& Vietri, M., 1986. MNRAS, $223,377$.

Gerhard, O.E., Vietri, M., \& Kent, S.M., 1989. ApJ, 345, L33.

Gilmore, G., King, I.R., \& van der Kruit, P.C., 1990. The Milky Way as a Galaxy, 19th Saas Fee Advanced Course, eds R. Buser \& I.R. King (Mill Valley: Univ. Science Books).

Glass, I., 1993. This volume.

Habing, H.J., 1988. A\&A, 200, 40.

Habing, H.J., Olnon, F.M., Winnberg, A., Matthews, H.E., \& Baud, B., 1983. A\&A, 128, 230.

Habing, H.J., Olnon, F.M., Chester, T., Gillett, F., Rowan-Robinson, M., \& Neugebauer, G., 1985. A\&A, 152, L1.

Harding, P., \& Morrison, H., 1993. This volume.

Harmon, R., \& Gilmore, G., 1988. MNRAS, 235, 1025.

Hauser, M.G., et al., 1990. NASA photograph G90-03046.

Hernquist, L., \& Weinberg, M.D., 1992. ApJ, 400, 80.

Hunter, C., \& Qian, E., 1993. MNRAS, in press.

Jarvis, B.J., \& Freeman, K.C., 1985. ApJ, 295, 324.

Jenkins, A., \& Binney, J.J., 1993. MNRAS, in press.

Kent, S.M., 1989. AJ, 97, 1614.

Kent, S.M., 1992. ApJ, 387, 181.

Kent, S.M., Dame, T.M., \& Fazio, G., 1991. ApJ, 378, 131.

Kinman, T.D., Feast, M.W., \& Lasker, B.M., 1988. AJ, 95, 804.

Kleinman, S.G., 1992. In Robotic Telescopes in the 1990s, ASP Conference Series No. 34, ed. A.V. Filippenko, p. 203.

Kuijken, K., \& Tremaine, S.D., 1991. In Dynamics of Disk Galaxies, ed. B. Sundelius (Göteborg, Sweden), p. 71.

Kuijken, K., \& Tremaine, S.D., 1993. ApJ, in press.

Lee, Y.-W., 1992. In IAU Symposium 149, The Stellar Populations of Galaxies, eds. B. Barbuy \& A. Renzini (Kluwer, Dordrecht), p. 446.

Le Poole, R.S., \& Habing, H.J., 1990. In Proceedings of the ESO/CTIO Workshop on Bulges of Galaxies, eds B.J. Jarvis \& D.M. Terndrup, p. 33.

Levison, H.F., \& Richstone, D.O., 1985a. ApJ, 295, 340.

Levison, H.F., \& Richstone, D.O., 1985b. ApJ, 295, 349.

Levison, H.F., \& Richstone, D.O., 1987. ApJ, 314, 476.

Lewis, J.R., \& Freeman, K.C., 1989, AJ, 97, 139.

Lindqvist, M., Habing, H.J., Winnberg, A., \& Matthews, H.E., 1992a. A\&AS, 92, 43.

Lindqvist, M., Habing, H.J., \& Winnberg, A, 1992b. A\&A, 259, 118.

Liszt, H.S., 1992. In The Center, Bulge, and Disk of the Milky Way, ed. L. Blitz, (Dordrecht: Kluwer), p. 111.

Liszt, H.S., \& Burton, W.B., 1980. ApJ, 236, 779.

Long, K., Ostriker, J.P., \& Aguilar, L., 1992. ApJ, 388, 362.

Matsumoto, T., Hayakawa, S., Koizumi, H., \& Murakawa, H., 1982. In The Galactic Centre, AIP Conf. 83, eds G.R. Riegler \& R.D. Blandford (New York: Am. Inst. of Physics), p. 48. 
McGinn, M.T., Sellgren, K., Becklin, E.E., \& Hall, D.N.B., 1989. ApJ, 338, 824.

Menzies, J.W., 1990. In Proceedings of the ESO/CTIO Workshop on Bulges of Galaxies, eds B.J. Jarvis \& D.M. Terndrup, p. 115.

Merrifield, M.R., 1991. AJ, $102,1335$.

Minniti, D., White, S.D.M., Olszewski, E.W., \& Hill, J.M., 1992. ApJ 393, L47.

Mould, J.R., 1983. ApJ, 266, 255.

Mulder, W.A., \& Liem, B.T., 1986. A\&A, 157, 148.

Nakada, Y., Deguchi, S., Hashimoto, O., Izumiura, H., Onaka, T., Sekiguchi, K., \& Yamamura, I., 1991. Nature, 353, 140.

Nakada, Y., Onaka, T., Yamamura, I., Deguchi, D., Ukita, N., \& Izumiura, H., 1993. PASJ, 45, in press.

Pfenniger, D., \& Norman, C., 1990. ApJ, 363, 391.

Pfenniger, D., \& Friedli, D., 1991. A\&A, 252, 75.

Raha, N., Sellwood, J.A., James, R.A., \& Kahn, F.D., 1991. Nature, 352, 411.

Rich, R.M., 1990. ApJ, 362, 604.

Richstone, D.O., 1980. ApJ, $238,103$.

Richstone, D.O., 1984. ApJ, 281, 100.

Rieke, G.H., \& Rieke, M.J., 1988. ApJL, 330, L33.

Rix, H.-W., \& White, S.D.M., 1992. MNRAS, 254, 389.

Rix, H.-W., Franx, M., Fisher, D., \& Illingworth, G.D., 1992. ApJL, 400, L5.

Rowley, G., 1988. ApJ, $331,124$.

Rybicki, G.R., 1987. In IAU Symposium 127, Structure and Dynamics of Elliptical Galaxies, ed. P.T. de Zeeuw (Dordrecht: Reidel), p. 397.

Satoh, C., 1980. PASJ, 32, 41.

Schwarzschild, M., 1979. ApJ, 232, 236.

Schwarzschild, M., 1993. ApJ, in press.

Sellgren, K., McGinn, M.T., Becklin, E.E., \& Hall, D.N.B., 1990. ApJ, 359, 112.

Sellwood, J.A., \& Sanders, R.H., 1988. MNRAS, 233, 611.

Sharples, R., Walker, A., \& Cropper, M., 1990. MNRAS, $246,54$.

Spaenhauer, A., Jones, B.F., \& Whitford, A.E., 1992. AJ, 103, 297.

Spergel, D.N., 1992. In The Center, Bulge, and Disk of the Milky Way, ed. L. Blitz (Dordrecht: Kluwer), p. 77.

Stark, A.A., 1977. ApJ, 213, 368.

Stasińska, G. 1993. This volume.

te Lintel-Hekkert, P., Dejonghe, H.B., \& Habing, H.J., 1991. Proc. Astron. Soc. Austr., 9, 20.

te Lintel-Hekkert, P., Versteege-Hensel, H.A., Habing, H.J., \& Wiertz, M., 1989. A\&AS, 78, 399.

te Lintel-Hekkert, P., Caswell, J.L., Habing, H.J., Norris, R.P., \& Haynes, R.F., 1991. A\&AS, 90, 327.

Toomre, A., 1982. ApJ, 259, 535.

Tyson, N.D., \& Rich, R.M., 1991. ApJ, 362, 547.

van Albada, G.D., 1985. In IAU Symposium 106, The Milky Way Galaxy, eds H. van Woerden, R.J. Allen, \& W.B. Burton (Dordrecht: Reidel), p. 547.

van der Marel, R.P., 1991. MNRAS, 253, 710.

van der Marel, R.P., \& Franx, M., 1993. ApJ, in press.

van Langevelde, H.J., 1992. PhD Thesis, Rijksuniversiteit Leiden.

van Langevelde, H.J., Frail, D.A., Cordes, J.M., \& Diamond, P.J., 1992a. ApJ, 396, 686.

van Langevelde, H.J., Brown, A.G.A., Lindqvist, M., Habing, H.J., \& de Zeeuw, P.T., 1992b. A\&AL, 261, L17.

Weinberg, M.D., 1992a. ApJ, 384, 81.

Weinberg, M.D., 1992b. ApJL, 392, L67.

Wesselink, Th., 1987. PhD Thesis, Nijmegen.

Whitelock, P.A., Feast, M.W., \& Catchpole, R.M., 1991. MNRAS, 248, 276.

Whitelock, P.A., \& Catchpole, R.M., 1992. In The Center, Bulge, and Disk of the Milky Way, ed. L. Blitz, (Dordrecht: Kluwer), p. 103.

Winnberg, A., Baud, B., Matthews, H.E., Habing, H.J., \& Olnon, F.M., 1985. ApJL, 291, L45.

Yuan, C., 1984. ApJ, 281, 600. 


\section{DISCUSSION}

Habing: Firstly, it is dangerous to immediately identify regression lines with a rotation curve, because there is also the distribution of the stars along the line of sight to be taken into account. Secondly, the IRAS data processing was a highly complex process and definitely non-linear at points. So, IRAS data in confused regions like the galactic plane, are beset by selection effects. Therefore an analysis, like that by Weinberg, can only be done by somebody with a relatively naive mind. Which may be an advantage sometimes, so I don't disagree with that approach, but I think it should not be believed until it has been confirmed by a completely independent study.

de Zeeuw: I agree, as you saw from the viewgraph.

Gerhard: Perhaps part of the confusion comes from trying to fit all observations into one coherent bulge picture. It may turn out that the disk itself has some interesting structure, in the inner kpc, which may be different from the structure of the Bulge. So it may not be the best to combine measurements from close to the plane and those well above the plane.

de Zeeuw: I agree, but all the matter moves in the same potential, so it would be nice to make one coherent picture.

Whitelock: Comparing the Lindquist OH/IR sample to that of te Lintel, which extends over the whole Bulge, it should be realised that the te Lintel sample is really somewhat biased towards the near side of the Bulge, due to sensitivity limitations in the Parkes telescope. I think that the Lindquist et al sample does not have this bias.

de Zeeuw: Yes, but I don't think that particular bias can explain the very different rotational kinematics between the two samples. But we should keep it in mind. 